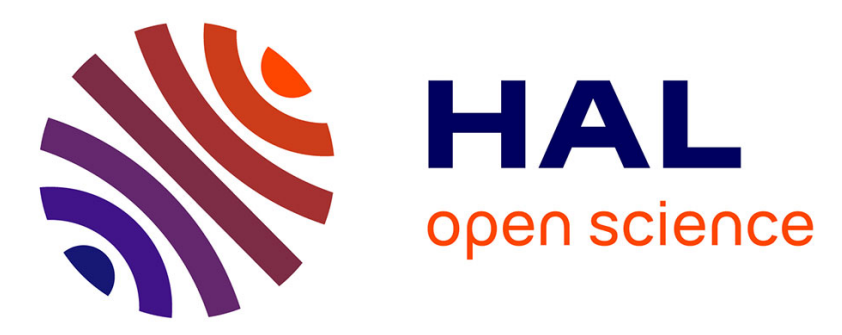

\title{
Étude d'un système de bombardement électronique de forte puissance en vue de la réalisation d'un ioniseur à champ magnétique axial
}

\author{
H. Lanteri, R. Bindi, G. Renucci
}

\section{To cite this version:}

H. Lanteri, R. Bindi, G. Renucci. Étude d'un système de bombardement électronique de forte puissance en vue de la réalisation d'un ioniseur à champ magnétique axial. Revue de Physique Appliquée, 1971, 6 (4), pp.425-432. 10.1051/rphysap:0197100604042500 . jpa-00243566

HAL Id: jpa-00243566

https://hal.science/jpa-00243566

Submitted on 1 Jan 1971

HAL is a multi-disciplinary open access archive for the deposit and dissemination of scientific research documents, whether they are published or not. The documents may come from teaching and research institutions in France or abroad, or from public or private research centers.
L'archive ouverte pluridisciplinaire HAL, est destinée au dépôt et à la diffusion de documents scientifiques de niveau recherche, publiés ou non, émanant des établissements d'enseignement et de recherche français ou étrangers, des laboratoires publics ou privés. 


\title{
ÉTUDE D'UN SYSTËME DE BOMBARDEMENT ÉLECTRONIQUE DE FORTE PUISSANCE EN VUE DE LA RÉALISATION D'UN IONISEUR A CHAMP MAGNÉTIQUE AXIAL
}

\author{
H. LANTERI, R. BINDI et G. RENUCCI
}

Faculté des Sciences de Nice, Laboratoire de Physique expérimentale

(Reçu le 13 octobre 1970, revisé le 14 juin 1971)

\begin{abstract}
Résumé. - Le présent article traite de la réalisation et des caractéristiques d'un système de bombardement électronique de forte puissance utilisant les principes du confinement magnétique du faisceau d'électrons, en vue de son application à l'ionisation d'nn jet atomique.

Cette méthode permet d'une part, d'augmenter le volume d'interaction entre le faisceau électronique et le jet atomique et d'autre part d'ioniser tout le volume du jet.
\end{abstract}

\begin{abstract}
The present paper deals with the realization and caracteristics of a high power electronic bombardment system using the principles of the magnetic confinement of the electronic beam ; the application of which is the ionization of atomic beams.

This system allows an increase of interaction volume between electronic and atomic beams, and the ionization of all the volume of the atomic beam.
\end{abstract}

I. Introduction. - Les systèmes d'ionisation de jets atomiques utilisent généralement un faisceau électronique perpendiculaire au jet atomique [1] à [20].

Toutefois, le module de la vitesse des atomes constituant le jet atomique est négligeable par rapport à celle des électrons, et le rendement d'ionisation est indépendant de l'angle sous lequel le faisceau électronique rencontre le jet atomique. Cette remarque conduit à effectuer le bombardement électronique dans le sens du jet atomique, en utilisant le principe du confinement magnétique.

F. Arvieux [21] a réalisé un tel système en utilisant un faisceau électronique tubulaire: le rendement d'injection (rapport du courant électronique utile lors de l'ionisation au courant électronique total) est de $27 \%$ pour une énergie de $1400 \mathrm{eV}$.

Afin d'augmenter le volume d'interaction entre le faisceau électronique et le jet atomique, et d'obtenir un faisceau électronique de forte puissance permettant l'ionisation d'un jet atomique cylindrique, plein, nous avons réalisé un canon du type Pierce [22], constitué par trois éléments.

L'étude de l'injection du faisceau d'électrons dans le champ magnétique axial fourni par un solénoïde permet de déterminer l'orientation optimale du canon à électrons.

II. Réalisation préliminaire d'un canon à électrons. - Les résultats obtenus lors de l'étude de l'influence de la forme de la cathode sur la répartition du potentiel entre cathode et anode ont conduit à la réalisation d'un canon dont une vue en section droite est donnée sur la figure 1. La partie émissive de la cathode est constituée soit par un ruban de tungstène, soit par une cathode à oxydes.

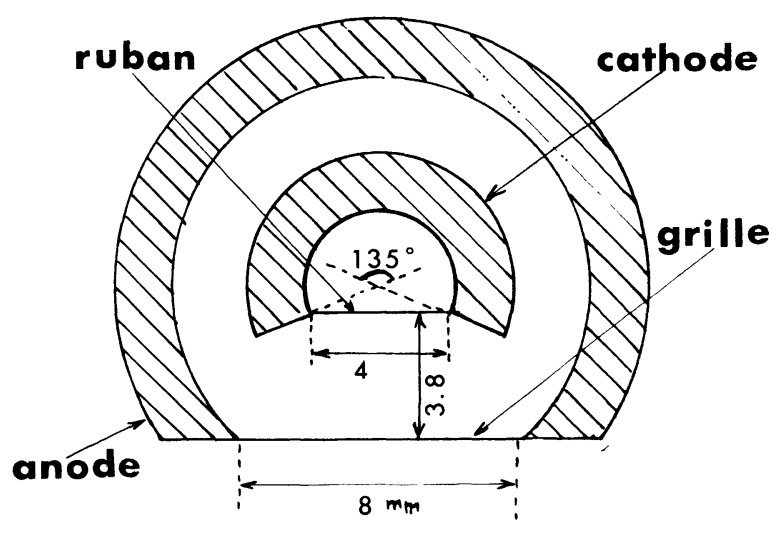

FIG. 1.

L'anode en acier inoxydable comporte une ouverture en travers de laquelle est placée une grille en tungstène de transparence égale à $95 \%$ permettant une répartition de potentiel convenable dans l'intervalle cathode-anode.

Lors de la détermination des caractéristiques du faisceau (intensité et géométrie), la cathode est portée à un potentiel négatif par rapport à l'anode. L'anode, ainsi qu'une cible recouverte de willémite permettant de visualiser l'impact du faisceau, sont au potentiel zéro.

Pour des tensions d'accélération comprises entre 
500 et 5000 volts, le faisceau électronique garde un angle d'ouverture constant et égal à $18^{\circ}$.

Toutes les mesures sont effectuées dans la gamme de pression $10^{-5}$ à $10^{-6}$ torr.

III. Etude de l'injection du faisceau d'électrons dans un champ magnétique. - Un système mécanique permet de modifier l'orientation du canon sans rompre le vide.

La figure 2 représente le positionnement optimal du canon à électrons tant pour la position de l'impact que pour le rendement d'injection.

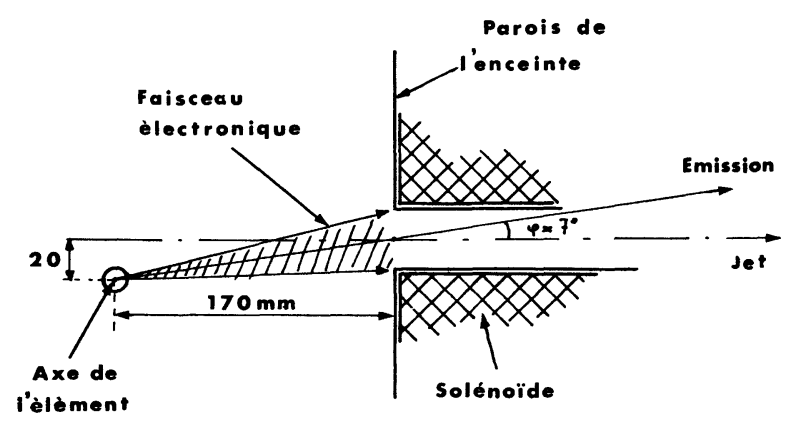

FIG. 2.

Cette position est indépendante de la tension d'accélération. L'intensité du faisceau au cours de ces mesures est inférieure à $20 \mathrm{~mA}$ et la tension d'accélération est comprise entre 500 volts et 2000 volts.

Le champ magnétique sensiblement uniforme sur une distance de $40 \mathrm{~cm}$, correspondant à la longueur de superposition du faisceau d'électrons et du jet atomique est obtenu à l'aide d'une bobine de 2700 spires.

L'impact est centré à $2 \mathrm{~mm}$ de l'axe du jet; cette position est indépendante du champ magnétique. La tache matérialisant l'impact a un diamètre de $2 \mathrm{~mm}$ pour un champ de 510 gauss : sa forme et sa grandeur restent inchangées lorsque nous déplaçons la cible le long du tube de confinement.

L'étude de l'influence du champ magnétique sur la forme de l'impact et sur l'intensité du courant collecté, lorsque la cible est placée au milieu du tube de confinement, conduit aux résultats suivants, indépendants de la position de la cible dans le tube de confinement.

Pour des valeurs du champ magnétique comprises entre 80 et 510 gauss, la tache reste sensiblement circulaire, son diamètre augmente lorsque le champ magnétique diminue. Par exemple, pour un champ de 340 gauss, l'impact a un diamètre de $6 \mathrm{~mm}$.

Pour des valeurs du champ magnétique inférieures à 80 gauss, nous notons une déformation importante de la forme de l'impact.

Le rendement d'injection a été mesuré sur une sonde de $55 \mathrm{~mm}$ de diamètre pour différentes valeurs de la tension d'accélération : les variations de ce rendement en fonction du champ magnétique pour une énergie des électrons de $1300 \mathrm{eV}$ et une intensité émise de $16 \mathrm{~mA}$ sont représentées sur la figure 3 .
Ces résultats ont conduit à la réalisation d'un canon de forte puissance constitué par trois éléments semblables au canon précédent, et occupant des positions symétriques autour de l'axe de l'enceinte.

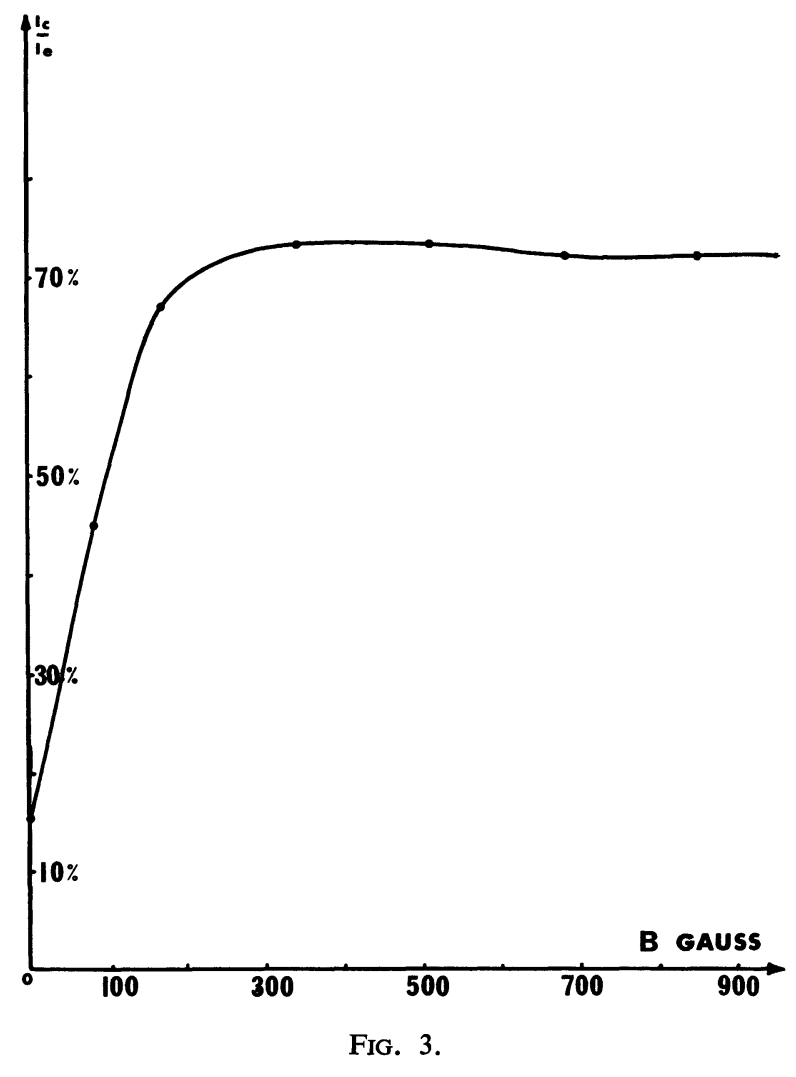

IV. Description du canon à électrons. - A) CATHODE. - Elle est de forme triangulaire et elle est fixée sur une plaque support en dural refroidie par circulation d'eau (Fig. 4). L'élément émissif est constitué par un ruban de tungstène de $2,5 \mathrm{~mm}$ de large.

Les différentes parties de la cathode peuvent se déplacer les unes par rapport aux autres de façon à maintenir les rubans de tungstène constamment tendus dans leur position initiale.

B) ANODE. - Elle épouse la forme de la cathode : elle est percée de trois ouvertures, situées en face des rubans émissifs, en travers desquelles est tendue une grille de tungstène dont la transparence est de $90 \%$.

L'ensemble du canon est représenté en coupe sur la figure 5.

V. Résultats expérimentaux. - Les performances de l'ioniseur étant essentiellement fonction des caractéristiques $\mathrm{du}$ faisceau électronique ionisant, nous avons étudié la proportion d'électrons émis, susceptible d'être utilisée lors de l'ionisation, ainsi que la répartition des intensités dans la zone d'interaction entre le faisceau électronique et le jet atomique, c'est-à-dire dans un cylindre de $2 \mathrm{~cm}$ de diamètre et de $40 \mathrm{~cm}$ de long autour de l'axe de la bobine de confinement et à l'intérieur de celle-ci. 


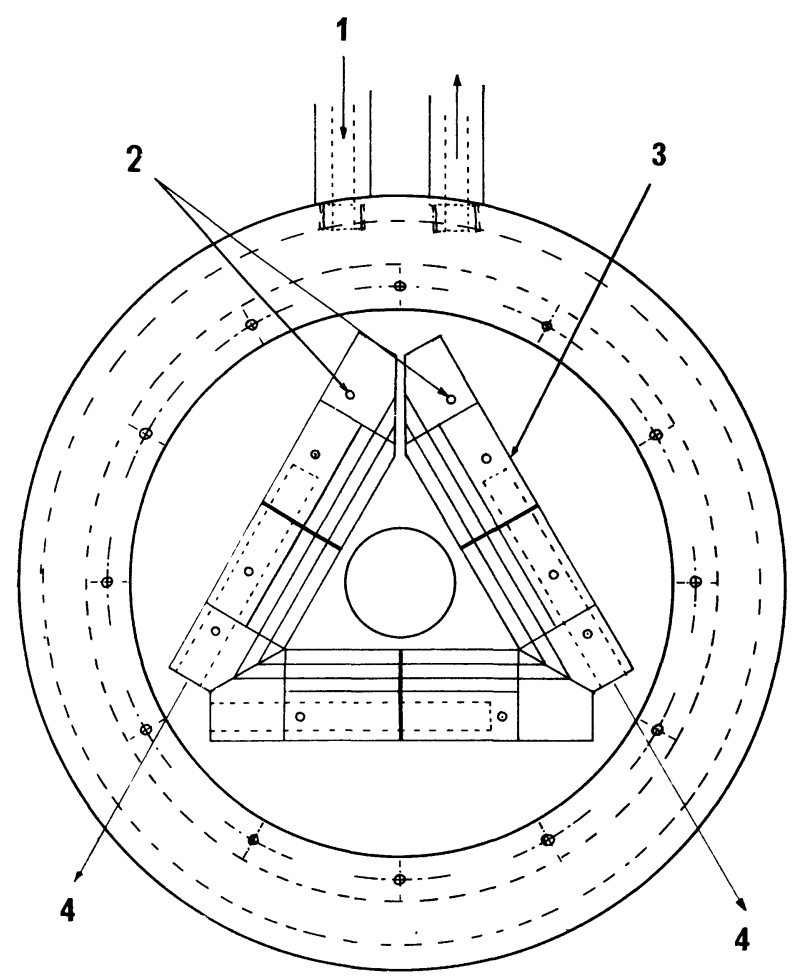

Fig. 4. - Position de la cathode sur son support refroidi : 1) Circulation d'eau. 2) Points d'alimentation de chauffage.

3) Cathode en 6 éléments. 4) Directions du déplacement.

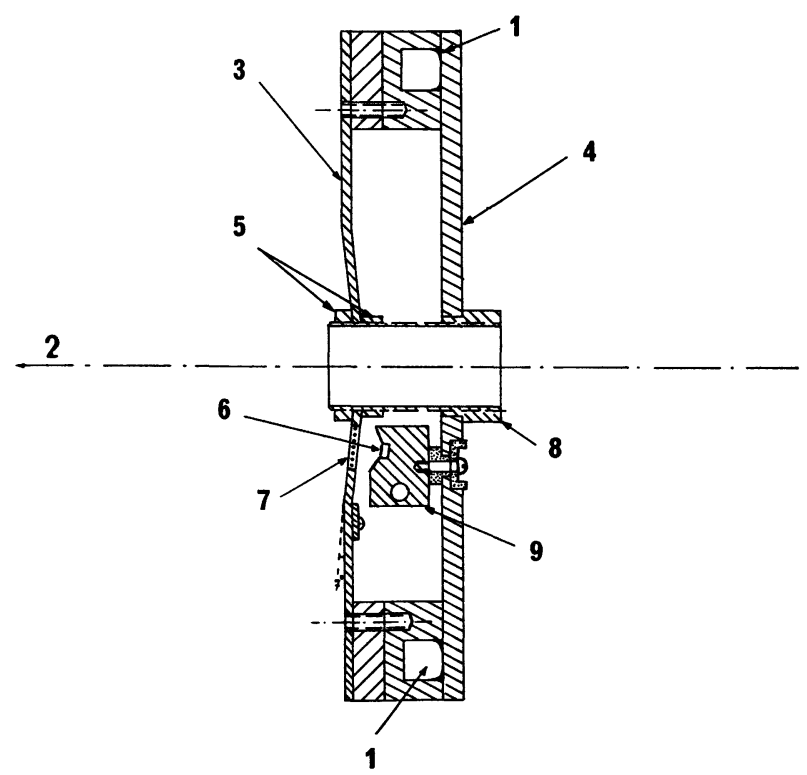

Fig. 5. - Vue en coupe du canon à électrons : 1) Circulation d'eau. 2) Direction du jet atomique. 3) Anode. 4) Plaque support. 5) Fixation de la grille. 6) Rubans émissifs. 7) Grille de tungstène. 8) Tube de centrage. 9) Cathode.

Les mesures du courant collecté ont été effectuées d'une part sur une sonde de $40 \mathrm{~mm}$ de diamètre afin de comparer les résultats avec ceux de l'étude préliminaire ; d'autre part sur une sonde de $20 \mathrm{~mm}$ de diamètre correspondant au diamètre de la zone d'interaction. Pour toutes ces mesures, les sondes sont placées au milieu du tube de confinement, car nous avons vérifié que les résultats étaient indépendants de la position de la cible dans le tube.

A) Courant collecté sur une sonde de $40 \mathrm{~mm}$ de diamètre : La sonde est portée au potentiel $V+24$ volts, $V$ étant le potentiel d'anode, pour éviter l'émission secondaire de la grille d'entrée de la sonde. Pour différentes valeurs de la température de la cathode comprises entre $1800^{\circ} \mathrm{K}$ et $2200^{\circ} \mathrm{K}$ nous avons tracé les courbes représentatives des variations du courant collecté $I$ en fonction de la valeur du champ magnétique de confinement $B$. Nous obtenons ainsi pour une température donnée de la cathode et pour diverses valeurs de $V$ comprises entre 0 et 1000 volts, un réseau de courbes analogue à celui représenté sur la figure 6 .

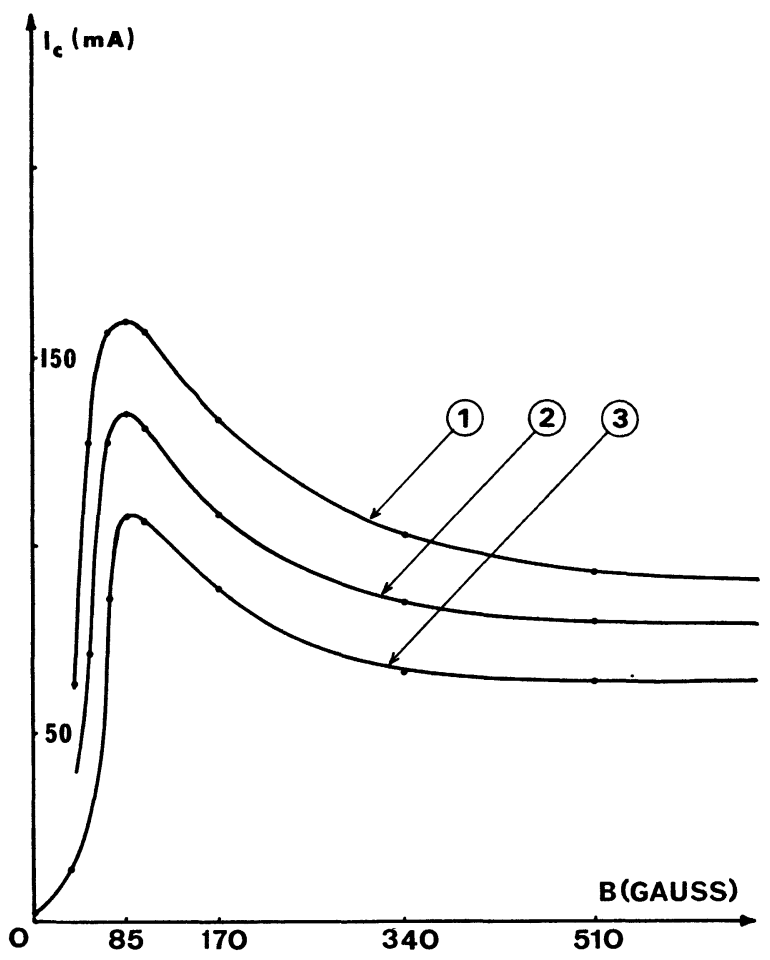

Fig. 6. - Courbes du courant collecté en fonction du champ magnétique sur une sonde de $40 \mathrm{~mm}$ de diamètre pour une température de cathode de $2100^{\circ} \mathrm{K}$ et pour différentes valeurs de la tension d'accélération : 1) $V=1000 \mathrm{~V}$; 2) $V=800 \mathrm{~V}$; 3) $V=500 \mathrm{~V}$.

Aux températures de la cathode pour lesquelles l'intensité émise est inférieure à $20 \mathrm{~mA}$, nous avons pu étendre le domaine des valeurs de la tension d'accélération jusqu'à 1800 volts ; figure 7 .

L'examen des divers réseaux de courbes montre l'existence d'un maximum très marqué pour une valeur du champ magnétique voisine de 85 gauss, quelles que soient les valeurs de la tension d'accélération et de la température de cathode.

La proportion d'électrons collectés au maximum d'intensité augmente avec la tension d'accélération ; pour un couple de valeurs $(B, V)$, cette proportion est, 


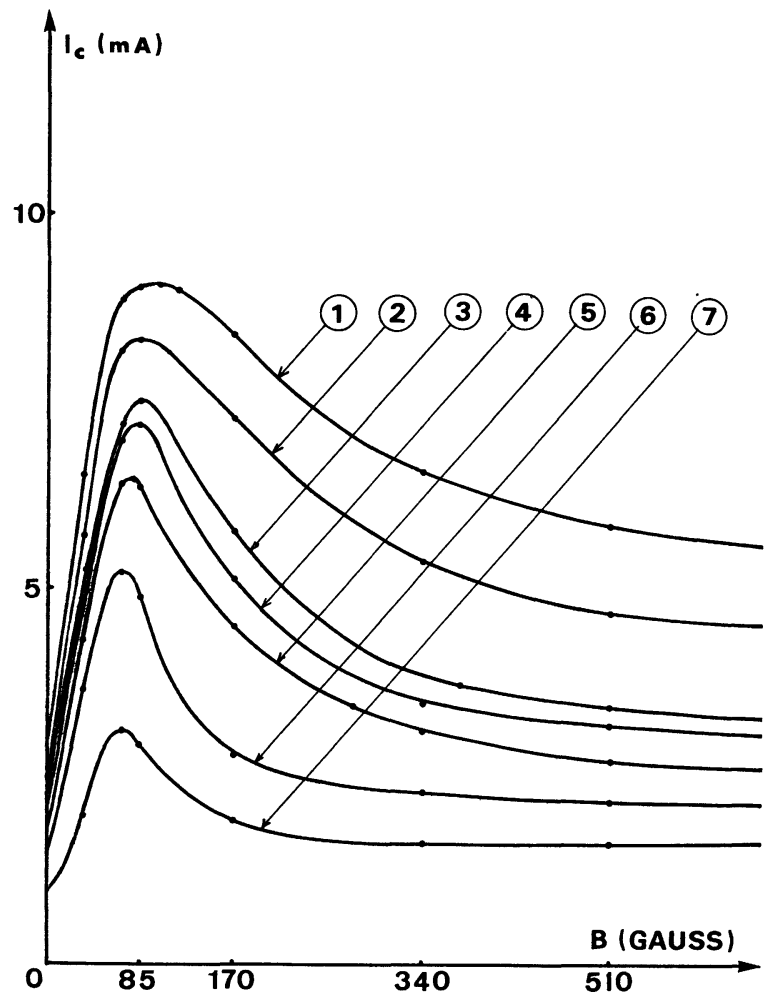

Fig. 7. - Courbes du courant collecté, en fonction du champ magnétique, sur une sonde de $40 \mathrm{~mm}$ de diamètre pour une température de cathode de $1850^{\circ} \mathrm{K}$ et pour différentes valeurs de la tension d'accélération : 1) $V=1800 \mathrm{~V}$; 2) $V=1400 \mathrm{~V}$; 3) $V=1000 \mathrm{~V}$; 4) $V=900 \mathrm{~V}$; 5) $V=700 \mathrm{~V}$; 6) $V=500 \mathrm{~V}$; 7) $V=300 \mathrm{~V}$.

aux erreurs de mesure près, indépendante de la température de la cathode, dans l'intervalle de température considéré.

Le tableau 1 indique les principaux résultats.

\section{TABLEAU 1}

$\begin{array}{lrrr}i_{\mathrm{c}} \text { (mA) } & >20 & <20 & <20 \\ V \text { (volts) } & 1000 & 1400 & 1800 \\ B \text { (gauss) } & 85 & 85 & 85\end{array}$

Rendement d'injection

$55 \% \quad 61 \% \quad 64 \%$

L'intensité maximum que nous avons pu obtenir est de $160 \mathrm{~mA}$ : elle correspond au cas de la figure 6 , pour lequel la température de la cathode est de $2100^{\circ} \mathrm{K}$.

Ces résultats conduisant à une variation importante du rendement d'injection avec le champ magnétique ne sont pas en accord avec ceux de l'étude préliminaire représentés sur la figure 3 et obtenus avec une sonde de $55 \mathrm{~mm}$.

Toutefois, l'examen du canon, après utilisation, a révélé des déformations de la grille dues à l'échauffement important résultant de l'utilisation de rubans de tungstène comme cathode. Lors de l'étude prélimi- naire, la cathode utilisée était une cathode à oxydes : dans ce cas, l'échauffement moins important n'avait pas entrainé de déformation notable de la grille. Ces déformations provoquent une divergence du faisceau qui n'est plus celle observée dans l'étude préliminaire. Pour un champ de 85 gauss, il semble que la plupart des électrons émis même s'ils ne sont pas injectés dans de bonnes conditions, soient collectés par la cible.

B) Courant collecté sur une sonde de $20 \mathrm{~mm}$ de diamètre : Le courant est mesuré sur une cible de $20 \mathrm{~mm}$ de diamètre enfermée dans une cage de Faraday et isolée de celle-ci : la cible et la cage de Faraday sont portées séparément à $V_{\mathrm{A}}+24 \mathrm{~V}$.

Les mesures doivent être majorées car $10 \%$ des électrons incidents sont interceptés par la grille d'entrée de la cage de Faraday.

Une étude identique à celle du paragraphe précédent conduit à des réseaux de courbes analogues à celui représenté sur la figure 8 qui correspond à une température de la cathode de $1850^{\circ} \mathrm{K}$.

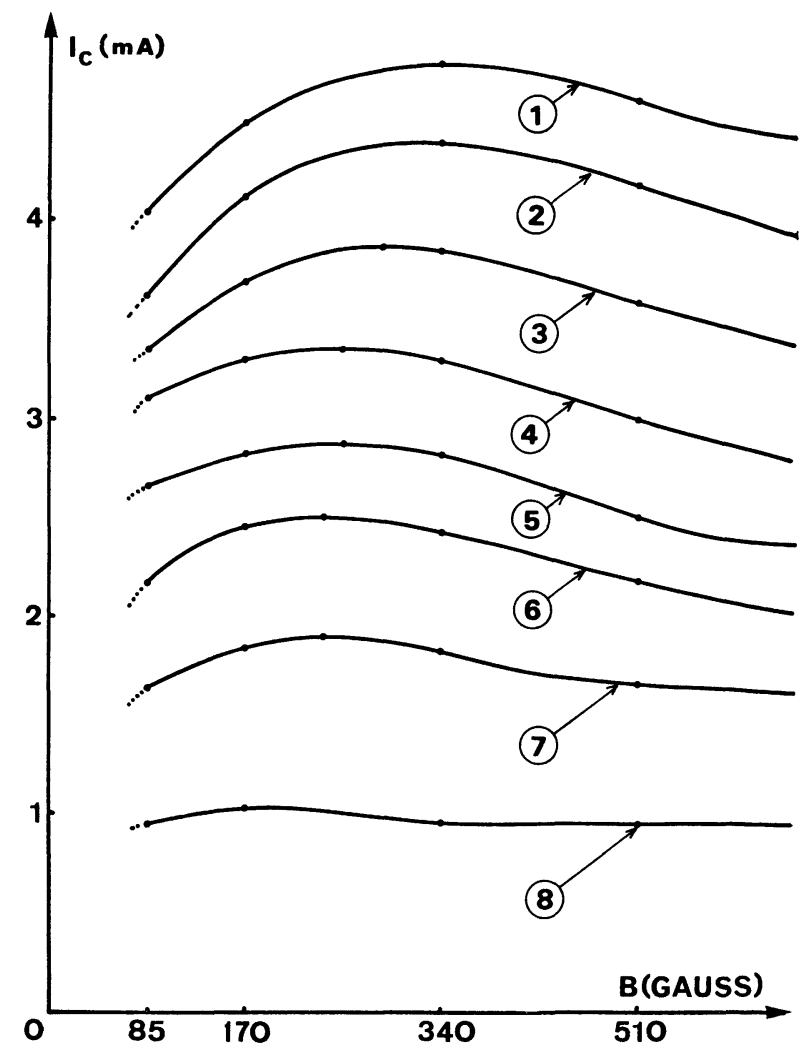

Fig. 8. - Courbes du courant collecté en fonction du champ magnétique sur une sonde de $20 \mathrm{~mm}$ de diamètre, pour une température de cathode de $1850^{\circ} \mathrm{K}$ et pour différentes valeurs de la tension d'accélération : 1) $V=1800 \mathrm{~V}$; 2) $V=1400 \mathrm{~V}$; 3) $V=1200 \mathrm{~V}$; 4) $V=1000 \mathrm{~V}$; 5) $V=800 \mathrm{~V}$; 6) $V=600 \mathrm{~V}$; 7) $V=400 \mathrm{~V}$; 8) $V=200 \mathrm{~V}$

Nous constatons, qu'en général, lorsque la valeur du champ magnétique est supérieure à 85 gauss, le rendement varie peu avec le champ magnétique.

La proportion d'électrons collectés reste sensiblement constante pour un couple de valeurs $(B, V)$, 
lorsque la température de la cathode varie dans l'intervalle considéré.

Par exemple, pour $V=1000$ volts et $B=170$ gauss, le rendement est égal à $27 \%$ compte tenu des corrections indiquées.

Pour des intensités émises inférieures à $20 \mathrm{~mA}$, le rendement d'injection passe à $38 \%$ pour une tension d'accélération de 1800 volts et un champ magnétique de 340 gauss.

L'intensité maximum obtenue a été de $75 \mathrm{~mA}$ pour un champ magnétique de 300 gauss environ et une tension d'accélération de 1000 volts.

Le réseau de courbes correspondant est représenté sur la figure 9.

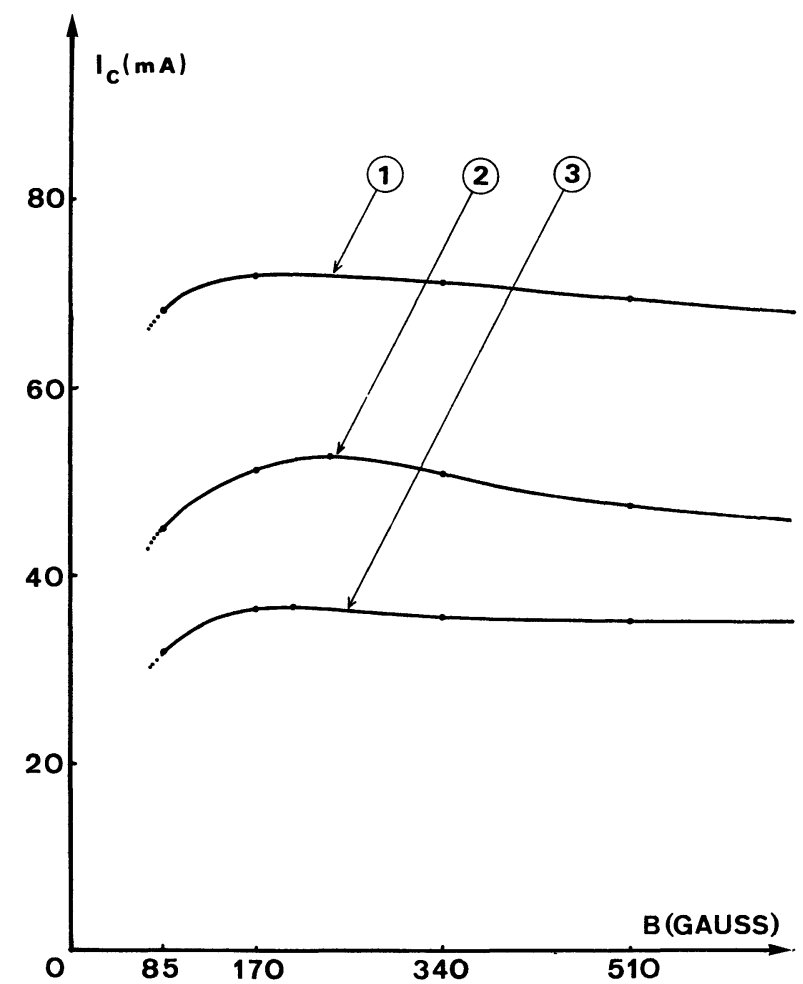

Fig. 9. - Courbes du courant collecté en fonction du champ magnétique sur une sonde de $20 \mathrm{~mm}$ de diamètre pour une température de cathode de $2100{ }^{\circ} \mathrm{K}$ et pour différentes valeurs de la tension d'accélération : 1) $V=1000 \mathrm{~V}$; 2) $V=800 \mathrm{~V}$; 3) $V=500 \mathrm{~V}$.

Ces résultats semblent confirmer la remarque faite au paragraphe précédent pour expliquer les différences observées entre les courbes du courant collecté sur une cible de $40 \mathrm{~mm}$ et les résultats obtenus dans l'étude préliminaire sur une cible de $55 \mathrm{~mm}$.

En effet, seuls arrivent sur la cible de $20 \mathrm{~mm}$ les électrons injectés dans le champ magnétique dans des conditions optimales, fixées lors de l'étude préliminaire, et ces électrons arrivent sur la cible quelle que soit la valeur du champ magnétique (pourvu qu'elle soit supérieure à 85 gauss). Une augmentation du champ magnétique n'entraîne qu'une diminution du diamètre de l'impact comme nous l'avons vu dans l'étude préliminaire.
Cette explication est aussi confirmée par la comparaison des courbes du rendement d'injection sur une cible de $55 \mathrm{~mm}$ (Fig. 3), de $40 \mathrm{~mm}$ (Fig. 7) et de $20 \mathrm{~mm}$ (Fig. 8).

En effet, nous constatons que les figures (3) et (8) présentent une allure identique, ce qui tendrait à prouver que les électrons qui atteignent la cible dans ces deux cas sont injectés dans le champ magnétique dans des conditions convenables.

Les différences dans les proportions observées semblent dues au fait que dans le cas de la sonde de $20 \mathrm{~mm}$ seule une partie des électrons est injectée convenablement dans le champ magnétique du fait de la déformation du canon à électrons.

C) Influence de la focalisation sur la forme du faisceau : Nous avons noté que pour des valeurs du champ magnétique comprises entre 0 et 85 gauss, le courant collecté présentait une suite de maximum et de minimum lorsque $B$ variait.

Ce phénomène est indiqué sur la figure 10 .

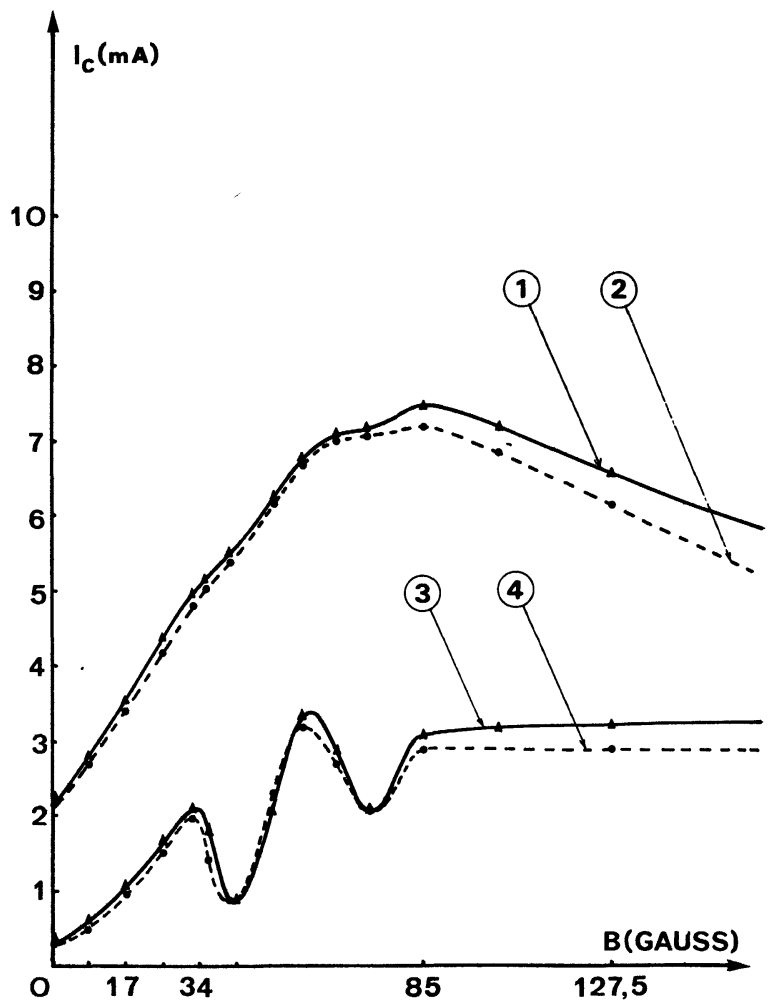

Fig. 10. - Courbes du courant collecté sur les deux types de sondes en fonction du champ magnétique compris entre 0 et 85 gauss. Température de cathode $1850^{\circ} \mathrm{K}$ :

- sonde de $40 \mathrm{~mm}$ de diamètre, tension d'accélération : 1) $V=1000 \mathrm{~V}$;2) $V=900 \mathrm{~V}$;

- sonde de $20 \mathrm{~mm}$ de diamètre, tension d'accélération :

3) $V=1000 \mathrm{~V}$; 4) $V=900 \mathrm{~V}$.

Cette courbe représente les variations du courant collecté soit sur une sonde de $20 \mathrm{~mm}$, soit sur une sonde de $40 \mathrm{~mm}$ de diamètre, pour des tensions d'ac- 
célération de 900 et 1000 volts, et pour une température donnée de la cathode.

Elle montre l'existence de deux maximum et de deux minimum.

Nous avons pu analyser ce phénomène à l'aide de photographies de la trace du faisceau d'électrons effectuées au cours de l'étude préliminaire. En effet, la cathode à oxydes utilisée en la circonstance provoque par dégazage une remontée de pression; cela nous a permis de visualiser le faisceau d'électrons qui ionisaient le gaz résiduel de l'enceinte à vide en donnant une luminescence bleutée. Nous avons pu voir ainsi l'effet des variations du champ magnétique sur la forme du faisceau.

Les photographies no 1 et $\mathrm{n}^{\circ} 2$ montrent, d'une part, l'existence de points de focalisation (foyers) où le faisceau est confiné dans un très petit domaine et de points de défocalisation où le faisceau a un diamètre beaucoup plus important; d'autre part, lorsque le champ magnétique augmente, les foyers reculent et sortent de la bobine, en même temps que le diamètre du faisceau diminue.

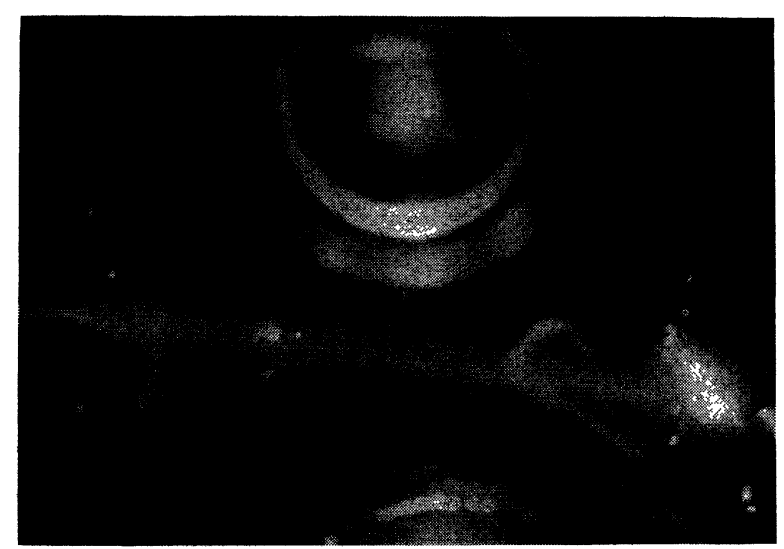

Photographie $1 B=340$ gauss.

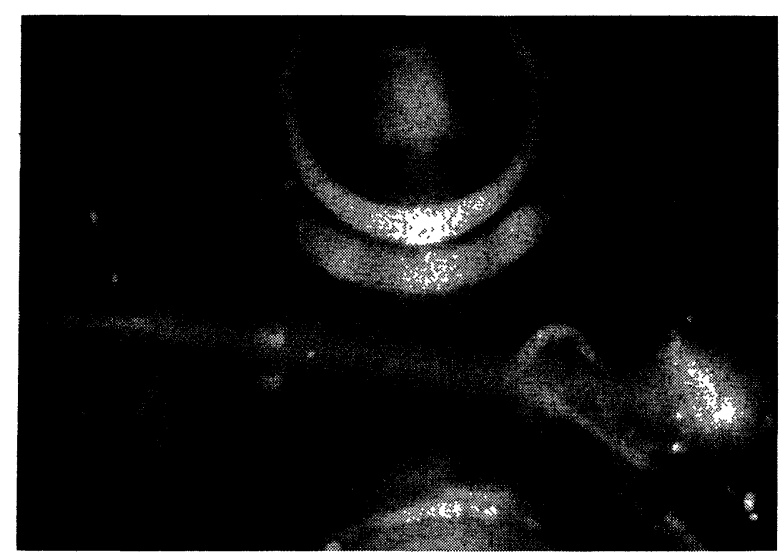

Photographie $2 B=680$ gauss.

Si maintenant la sonde de mesure est située au milieu de la bobine de confinement, lorsque le champ magnétique augmente, le foyer recule et arrive au niveau de la sonde, nous observons un maximum d'intensité, puis si le champ augmente, le foyer continue à reculer et nous observons un premier minimum lorsqu'un point de défocalisation arrive au niveau de la sonde : il en sera de même pour le maximum et le minimum suivants.

Lorsque le diamètre du faisceau est inférieur à $20 \mathrm{~mm}$ au niveau des points de défocalisation, les variations ne se font plus sentir : le courant reste alors sensiblement constant.

Nous attribuons cet effet au champ magnétique car dans l'étude préliminaire nous n'avons noté aucun phénomène de focalisation lors de l'étude de la forme du faisceau.

Ces résultats sont confirmés par les mesures plus précises du courant recueilli sur la sonde de $40 \mathrm{~mm}$ qui présente des variations d'intensité correspondant aux mêmes valeurs du champ magnétique que précédemment.

D) Topographie du faisceau électronique ;

Pour terminer l'étude du faisceau électronique, nous avons tenté de donner une topographie de ce faisceau à l'aide d'une sonde excentrée dont le diamètre de l'orifice d'entrée a été calibré à $4 \mathrm{~mm}$.

Les déplacements de la sonde sont repérés de la façon suivante (Fig. 11). Nous avons tracé des topographies pour différentes températures de la cathode.

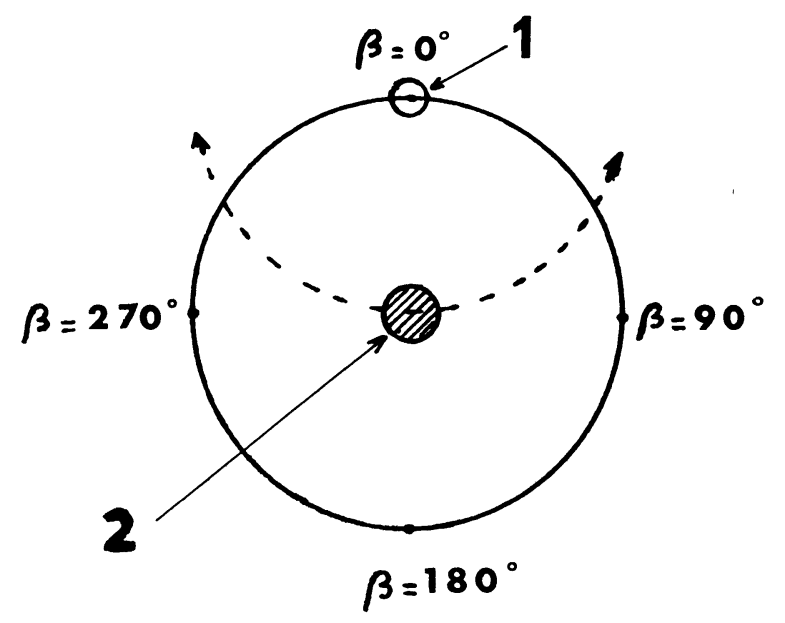

FIG. 11. - Repérage de la sonde : 1) Axe de la sonde ; 2) Orifice de collection.

Les courbes obtenues sont semblables à celles représentées sur les figures suivantes, qui correspondent à des températures de cathode de 2080 et $2100^{\circ} \mathrm{K}$ (Fig. 12, 13).

Le résultat essentiel est le fait qu'un courant d'électrons relativement important atteint le centre de la cible puisque pour un champ magnétique de 170 gauss et une tension d'accélération de 1000 volts, la propor- 


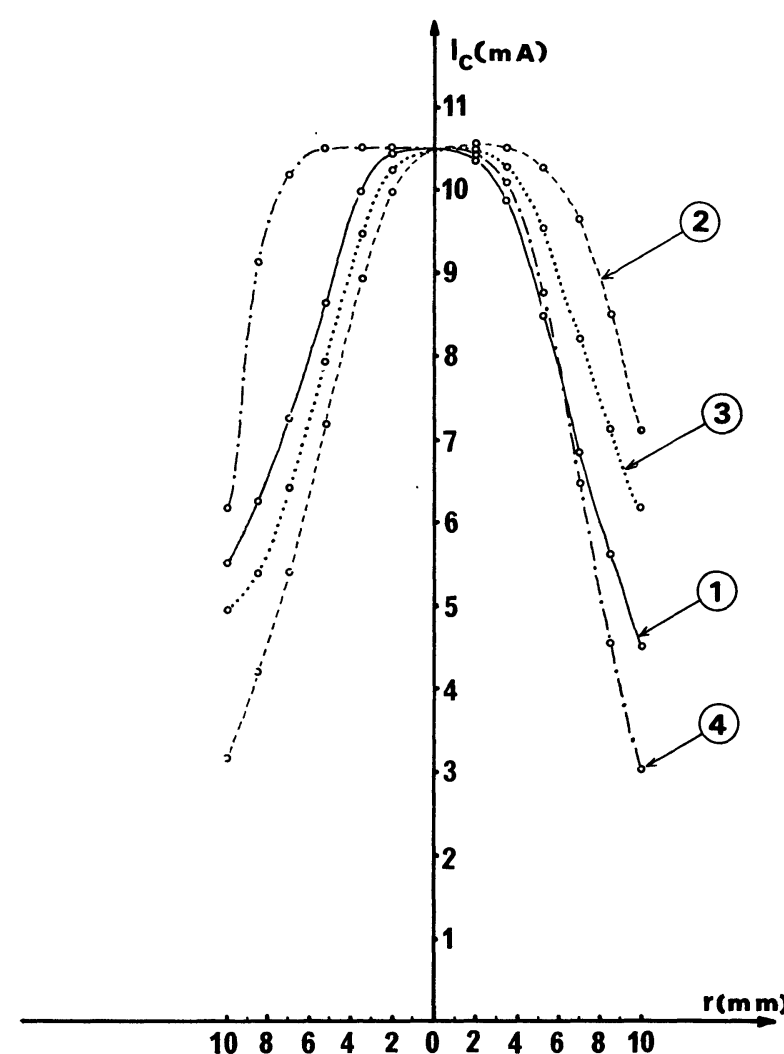

Fig. 12. - Topographie du faisceau électronique au milieu du tube de confinement pour $T^{0}=2080^{\circ} \mathrm{K}, V=1000 \mathrm{~V}$; $B=170$ gauss ; 1) $\beta=0^{\circ}$; 2) $\beta=90^{\circ}$; 3) $\beta=180^{\circ}$; 4) $\beta=270^{\circ}$.

tion d'électrons collectés sur la cible de $4 \mathrm{~mm}$ placée sur l'axe de l'enceinte, est de 3,5\% c'est-à-dire plus importante en moyenne que dans le reste de la section droite du faisceau.

Nous avons remarqué que cette proportion restait constante lorsque la température de la cathode variait.

VI. Conclusion. - L'appareil que nous avons construit de façon à ne pas intercepter le jet atomique, permet d'obtenir un courant électronique de $75 \mathrm{~mA}$ pour une énergie de 1000 électron-volts dans des conditions telles que tout le volume d'ionisation soit utilisé ; ce qui, à notre connaissance, n'était pas le cas jusqu'à présent

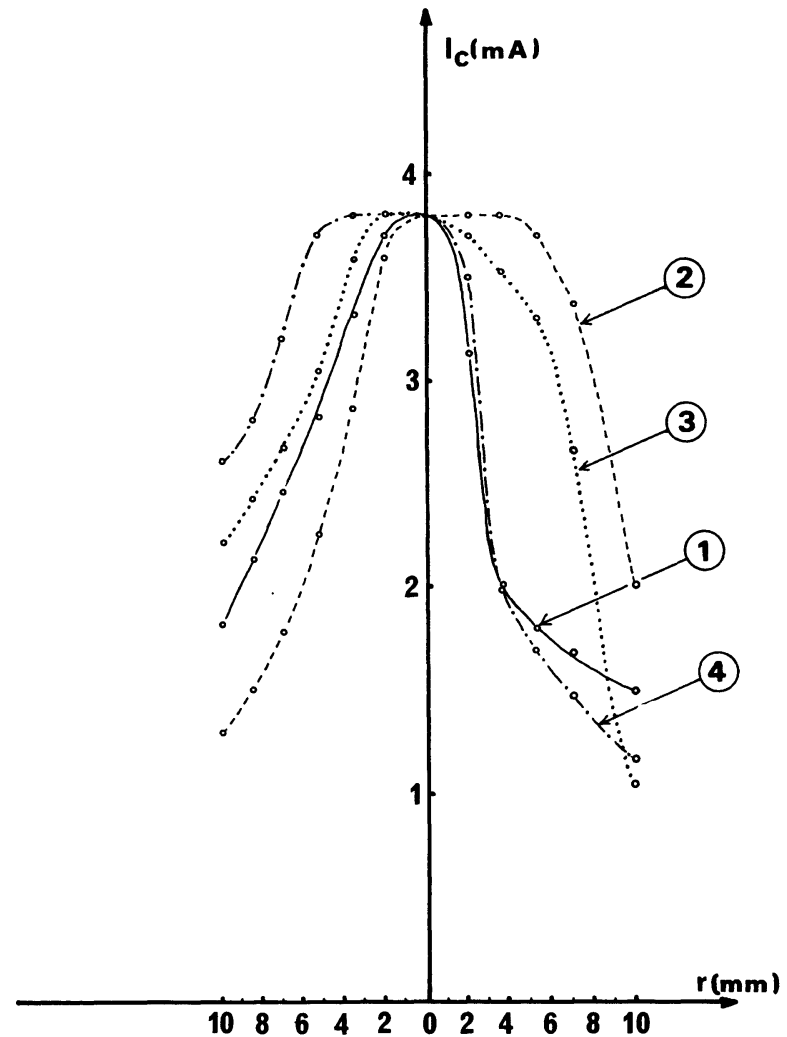

FIG. 13. - Topographie du faisceau électronique au milieu du tube de confinement pour $T^{0}=2100^{\circ} \mathrm{K} ; V=1000 \mathrm{~V}$; $B=170$ gauss ; 1) $\beta=0^{\circ}$; 2) $\beta=90^{\circ}$; 3) $\beta=180^{\circ}$; 4) $\beta=270^{\circ}$

Le rendement d'injection obtenu est supérieur à celui obtenu jusqu'ici puisqu'il est de $27 \%$ à une énergie de $1000 \mathrm{eV}$. De plus, les résultats obtenus úissent espérer un rendement d'injection de $38 \%$ pour une énergie de $1800 \mathrm{eV}$.

Enfin, lors de l'étude préliminaire nous avons obtenu un rendement d'injection de $72 \%$ pour une énergie de $1300 \mathrm{eV}$ et des rendements équivalents pourraient donc être obtenus avec un canon de forte puissance dans la mesure où les conditions géométriques fixées dans l'étude préliminaire sont respectées ce qui doit être possible en utilisant une cathode fournissant une intensité élevée pour une température plus basse que celle utilisée ici.

\section{Bibliographie}

[1] Beurtey-Thirion-Papineau, Sources de protons et de deutons polarisés, Nuovo cim., 1961, 19, 207.

[2] Dickson (J. M.), Polarized ion sources and acceleration of polarized beams; Progress in nuclear techniques and instrumentation, 1964, I, 105.

[3] Craddock (M. K.), Helv. Phys. Acta Suppl., 1960, VI, 59-67.

[4] Collins (E. R.), Glasvish (H. F.), Whineray (S.), Nuclear instruments and methods, 1963, 25, 67-76.
[5] Fite (W. L.), Brackmann (R. T.), Phys. Rev., 1958, 112, 1141-1151.

[6] Planiol (R.), C. R. Acad. Sci. Paris, 1935, 539, 200.

[7] Clausnitzer (G.), Nuclear instruments and methods, 1963, 23, 309.

[8] Clausnitzer (G.), Helv. Phys. Acta. Suppl., 1960, VI, 35.

[9] Sampson (M. B.), Bleakney (W.), Phys. Rev., 1936, 732-50. 
[10] Smith (L. P.), Carlock (A. H.), Phys. Rev., 1936, 646-59.

[11] Wessel (G.), Lew (H.), Phys. Rev., 1953, 641-92.

[12] Bloch (F.), Phys. Rev., 1946, 70-640.

[13] Abragam (A.), Phys. Rev. Letters, 1958, 1, 374

[14] Sherwood (J. E.), Bull. Ann. Phys. Soc., 1956, 11 161

[15] Sherwood (J. E.), Bull. Ann. Phys. Soc., 1960, 5, 441.

[16] Sherwood (J. E.), Nuclear Inst. and Methods, 1961, $6,307$.
[17] SHERwood (J. E.), Nuclear Inst. and Methods, 1962, $15,103$.

[18] Ripouteau (F.), Etude bibliographique, Laboratoire de Physique Nucléaire de Grenoble. Sept., 1962.

[19] KelleR (R.), Rapport C. E. R. N., 57-30.

[20] Keller (R.), Rapport C. E. R. N., 60-02.

[21] Arvieux (F.), Thèse de $3^{3}$ cycle Grenoble, 1964.

[22] Pierce (J. R.), Theory and design of electron beams Van Nostrand, 1954. 